\section{SOI: $1.1 /$ TAS DOI: $10.15863 /$ TAS International Scientific Journal Theoretical \& Applied Science}

p-ISSN: 2308-4944 (print) e-ISSN: 2409-0085 (online)

Year: 2018 Issue: $06 \quad$ Volume: 62

Published: $25.06 .2018 \quad$ http://T-Science.org
Dokturbek Adambekovich Adambekov Doctor of medical sciences, head of microbiology department of KSMA, Bishkek city, Kyrgyz Republic

Asel Dokturbekovna Adambekova Candidate of medical sciences, assistant professor of microbiology department of KRSU, Bishkek city, Kyrgyz Republic

Abdullat Samatovich Kadyrov

Doctor of medical sciences, head of National center of phthisiology

\title{
IMPLEMENTATION OF THE XPERT MTB / RIF ASSAY IN THE KYRGYZ REPUBLIC, RESULTS, LESSONS, FUTURE PROSPECTIVE
}

Abstract: On the basis of these results we can conclude that the use of the test Xpert MTB / RIF gives good results in routine use to identify people with $T B$ and MDR-TB. But along with this, further study of cases of discrepancies in the results for the detection of resistance to rifampicin.

Key words: Xpert MTB/RIF assay, M. tuberculosis, resistance, rifampicin.

Language: Russian

Citation: Adambekov DA, Adambekova AD, Kadyrov AS (2018) IMPLEMENTATION OF THE XPERT MTB / RIF ASSAY IN THE KYRGYZ REPUBLIC, RESULTS, LESSONS, FUTURE PROSPECTIVE. ISJ Theoretical \& Applied Science, 06 (62): 52-55.

Soi: http://s-o-i.org/1.1/TAS-06-62-12 Doi: crossef https://dx.doi.org/10.15863/TAS.2018.06.62.12

\section{ИМПЛЕМЕНТАЦИЯ ТЕСТА ХРЕRТ МТВ / RIF В КЫРГЫЗСКОЙ РЕСПУБЛИКЕ, РЕЗУЛЬТАТЫ, УРОКИ, ДАЛЬНЕЙШЕЕ РАЗВИТИЕ}

Аннотация: Исходя, из представленных результатов можно сделать выводы, что использование теста Хреrt MTB/RIF дает хорошие результаты при рутинном использовании для выявления случаев с ТБ и МЛУ ТБ. Но наряду с этим, необходимо дальнейшее изучение случаев расхождения результатов по выявлению устойчивости к рифампицину.

Ключевые слова: анализ Xpert MTB/RIF, M. tuberculosis, yстойчивость к рифампицину.

\section{Введение}

Согласно оценке Всемирной Организации Здравоохранения (ВО3) Кыргызская республика входит в число 27 стран с наиболее высокими показателями распространенности лекарственноустойчивых форм туберкулёза [1]. Туберкулез с множественной лекарственной устойчивостью (МЛУ ТБ) остается серьезной угрозой системе общественного здравоохранения [2]. По оценкам ВО3, заболеваемость туберкулезом в 2013 году составила 101 случаев на 100000 населения вместе с пенитенциарной системой [3]. В 2011 году, доля МЛУ-ТБ среди новых случаев заболевания была $26,0 \%$, а среди ранее леченных случаев 52,0\% [4].

В 2010 г. ВО3 рекомендует использование теста Xpert MTB / RIF, быстрого молекулярного диагностического теста, который выявляет наличие ДНК комплекса МТБ, а также устойчивость к рифампицину [5]. С 2010 года, ряд стран, включая Кыргызстан внедрили использование Xpert MTB / RIF в алгоритм исследования лиц с подозрением на ТБ [6]. В большинстве стран имплементация Xpert MTB / RIF была проанализирована и получены данные об эффективности теста. Выводы, полученные в ходе многоцентровых исследований, включая такие страны, как Южная Африка, Перу, Индия, Азербайджан, Уганда и Республики Филиппины продемонстрировали хорошую производительность теста Xpert MTB / RIF [7]. Точность Xpert MTB / RIF, его чувствительность и специфичность для выявления пациентов с ТБ, МЛУ ТБ или ВИЧ-ассоциированного ТБ, также была оценена и представлена в обзоре Кохрейна [8]. Национальная программа по борьбе с туберкулезом координирует имплементацию нового метода и осуществляет сбор данных из лабораторий.

Материалы и методы: Данные, используемые в настоящем исследовании, были получены из восьми лабораторий, где расположены платформы GentXpert, также и Национальной референс-лаборатории с июля 
2012 года по декабрь 2014. Это ретроспективное исследование проанализировало рутинные результаты лабораторного исследования лиц с подозрением на ТБ согласно национальному алгоритму [9].

Проведена сравнительный анализ характеристик теста Xpert MTB/RIF и традиционных, культуральных методов исследования на автоматизированной системе BACTEC MGIT 960 и среде ЛевенштейнаЙенсена.

Целью данного исследования был анализ результатов имплементации теста Xpert MTB/RIF в Кыргызстане, оценка эффективности теста по сравнению с традиционными методами исследования.

\section{Статистическая обработка данных}

Чувствительность и специфичность теста Xpert MTB/RIF были рассчитаны по сравнению с автоматизированной системой ВАСТЕC MGIT 960 и посевами на плотной, яичной среде Левенштейна-Йенсена, используемых как золотой стандарт. Статистическая обработка была выполнена на программном обеспечении STATA, версия 14.0 и Excel 2010.

Результаты и их обсуждение

В исследование были включены результаты исследований у $2,743(60,2 \%)$ мужчин и 1,817 (39.8) женщин, средний возраст которых составил 36 лет. Было проведено 5,612 лабораторных исследований на Xpert MTB/RIF, GenoType MTBDR plus, v.2.0, BACTEC MGIT 960 и посевы на среде ЛЙ (табл. 1).

\section{Количество проведенных лабораторных исследований на ТБ}

Таблица 1.

\begin{tabular}{|l|c|c|}
\hline \multicolumn{1}{|c|}{ Наименование тестов } & $\begin{array}{c}\text { Комплекс МТВ } \\
\text { (выявлен/не выявлен), } \mathbf{n}\end{array}$ & $\begin{array}{c}\text { Устойчивость к Rif } \\
\text { (выявлена/не выявлена), } \mathbf{n}\end{array}$ \\
\hline Xpert MTB/RIF & 2,217 & 1,165 \\
\hline GenoType MTBDR plus, v.2.0 & 661 & 387 \\
\hline ВАСТЕС MGIT 960 & 1554 & 414 \\
\hline Среда Левенштейна-Йенсена & 1180 & 364 \\
\hline Всего & $\mathbf{5 , 6 1 2}$ & $\mathbf{2 , 3 3 0}$ \\
\hline
\end{tabular}

Сравнительный анализ тестов Xpert MTB/RIF и GenoType MTBDR plus,v.2.0, выявил, что 530 из 559 дали положительный результат на наличие ДНК комплекса МБТ при тестировании обеими методами (93,9\%). Что касается выявления комплекса МТБ, 91 из 102 результатов испытаний были отрицательными $(89,2 \%)$.

Такие параметры теста, как чувствительность и специфичность были проанализированы по сравнению с ВАСТЕС MGIT 960 и исследованиями на среде Левенштейна-Йенсена. По сравнению с ВАСТЕС MGIT 960 Xpert MTB/RIF был положительным в 922 случаях из $1000 \quad(92.2 \%)$ и 499 отрицательных результата из 554 штаммов
(90.1\%). На среде Левенштейна-Йенсена чувствительность теста составила $93.4 \%$ (655/701) и специфичность 90.8\% (435/479). Расхождение результатов Xpert и MGIT составила 8.5\% (133/1554), a Xpert MTB/RIF и ЛЙ - 7.6\% (90/1180).

Относительно исследования штаммов на лекарственную чувствительность к рифампицину, 2,330 образцов тестированные Xpert также были подвергнуты анализу либо на GenoType MTBDR plus, либо на MGIT и ЛЙ. В 131 случае из 150 Xpert положительный и рифамицин устойчивый результат совпали с GenoType MTBDR plus (87.3\%) и 220 из 237 были с чувствительностью к рифампицину $(92.8 \%)$.

\section{Сравнение Хpert MTB/RIF с MGIT и ЛЙ}

Таблица 2.

\begin{tabular}{|ll|l|l|l|l|}
\hline & $\begin{array}{l}\text { Всего } \\
\text { посевов, }\end{array}$ & Чувствительность \% (n) & Спцифичность\% (n) & $\begin{array}{l}\text { Совпадение } \\
\text { результатов \% (n) }\end{array}$ \\
\hline $\begin{array}{l}\text { Посев } \\
\text { МGIT на }\end{array}$ & 1554 & $92.2 \%(922 / 1000)$ & $90.0 \%(499 / 554)$ & $91.4 \%(1421 / 1554)$ \\
\hline $\begin{array}{l}\text { Посев на } \\
\text { ЛЙ }\end{array}$ & 1180 & $93.4 \%(655 / 701)$ & $90.8 \%(435 / 479)$ & $92.4 \%(1090 / 1180)$ \\
\hline
\end{tabular}


Сравнение Xpert и посева на среду ЛЙ показали чувствительность 90.9\% (120/132) и специфичность 91.4\% (212/232). В случае сравнения с ВАСТEC MGIT 960, Xpert показал чувствительность $\quad 89.3 \% \quad(108 / 121)$ и специфичность 90.1\% (264/293) (табл. 2).
Пропорция расхождений результатов по рифампицину между Xpert и GenoType MTBDR plus составила $6.05 \%$ (40/661) от всех результатов ТЛЧ, в то же время расхождение между Xpert и MGIT составила $8.5 \%(133 / 1554)$, а Xpert и LJ $7.6 \%(90 / 1180)$.

Таблица 3.

Сравнение результатов различных методов по выявлению устойчивости к рифампицину

\begin{tabular}{|l|c|c|c|}
\hline \multirow{2}{*}{} & \multicolumn{2}{|c|}{ Хреrt MTB/RIF } & \\
\cline { 2 - 4 } & Rif-устойчивый & Rif-чувствительный & Совпадение результатов (\%) \\
\hline MGIT & $89 \%(108 / 121)$ & $90 \%(264 / 293)$ & $90 \%(372 / 414)$ \\
$95 \%$ ДИ - \% & $82.4-93.6$ & $86.1-93.0$ & $86.5-92.4$ \\
\hline ЛЙ & $91 \%(120 / 132)$ & $91 \%(212 / 232)$ & $91 \%(332 / 364)$ \\
\hline & & & $87.8-93.7$ \\
\hline
\end{tabular}

\section{Обсуждение результатов}

В данном исследовании были оценены результаты имплементации Xpert MTB/RIF в рутинном применении для выявления лиц, болеющих ТБ в Кыргызской Республике. Результаты нашего исследования показали чувствительность теста Xpert MTB/RIFдля выявления комплекса МБТ по сравнению с BACTEC MGIT 960 - 91,4\% и по сравнению с использованием среды ЛЙ - 92,4\%. Эты данные выше, чем было представлено Marlowe EM и авт. [10]. Также, было выявлено высокое совпадение результаов Xpert MTB/Rif и GenoType MTB DR plus - 94\%. Так ка в направлениях от клиницистов не было указание наличие подозрения на МЛУ ТБ, в нашем исследовании не оказалось возможным рассчитать чувствительность и специфичность по данном параметру как у Xichao Ou и авт [11]. Точно так же, стратификация пациентов по их истории предыдущего лечения туберкулеза было бы очень полезно в выявлении большего количества ложно-положительных результаты, как предложено Boyles ТН и авт. [12].

Совпадение результатов ТЛЧ между Xpert MTB/RIF и фенотипическими исследованиями на жидких и плотных средах составило 90\% и 91\% соответственно. Данный факт совпадает с результатами исследования, проведенного в Индонезии [13, 14, 15], где было выявлено совпадение результатов в $89,3 \%$.

В проведенном исследовании, в лаборатории, где проводиться Xpert MTB/RIF и традиционные методы выявления ТБ доставляются разные пробы патологического материла, что может также приводит к несовпадению результатов, общая доля которых составила менее 10\% (110/1070). О случаях "ложной" резистентности к РИФ, каковые мы наблюдали в нашем исследовании, также сообщалось в ряде исследований $[16,17,18,19]$. Также нами выявлено, что $32,7 \%$ (36/110) образцов, которые показали, различные результаты были доставлены в лаборатории с задержкой в 4-10 дней. Вышесказанное свидетельствует о необходимости более углубленного изучения случаев расхождения результатов молекулярно-генетического метода и традиционных, в будущем. Необходимо проведение секвенирования образцов мокроты, также более тщательное изучение истории предыдущего лечения ТБ больных.

Учитывая простоту технологии, проведение тестирования Xpert MTB/RIF имеет большие преимущества особенно в сельских районах, где не налажена система транспортировка образцов мокроты, нестабильное электроснабжение.

Дальнейшее исследование необходимо для углубленного анализа результатов с расхождениями с устойчивостью к рифампицину путем проведения секвенирования ДНК, что поможет разъяснить природу расхождений.

\section{Заключение}

Исходя, из представленных результатов можно сделать выводы, что использование теста Xpert MTB/RIF дает хорошие результаты при рутинном использовании для выявления лиц с ТБ и МЛУ ТБ. Но наряду с этим, необходимо дальнейшее изучение случаев расхождения результатов по выявлению устойчивости к рифампицину. 


\begin{tabular}{l|lr|ll|ll} 
& ISRA (India) & $=\mathbf{1 . 3 4 4}$ & SIS (USA) & $=\mathbf{0 . 9 1 2}$ & ICV (Poland) & $=\mathbf{6 . 6 3 0}$ \\
Impact Factor: & ISI (Dubai, UAE) $=\mathbf{0 . 8 2 9}$ & PUHЦ (Russia) $=\mathbf{0 . 2 0 7}$ & PIF (India) & $=\mathbf{1 . 9 4 0}$ \\
& GIF (Australia) & $\mathbf{0 . 5 6 4}$ & ESJI (KZ) & $=\mathbf{4 . 1 0 2}$ & IBI (India) & $=\mathbf{4 . 2 6 0}$ \\
& JIF & $=\mathbf{1 . 5 0 0}$ & SJIF (Morocco) & $=\mathbf{2 . 0 3 1}$ & & \\
\hline
\end{tabular}

\section{References:}

1. (2018) UN Data. World Statistics Pocketbook. Country profile: Kyrgyzstan. https://data.un.org/CountryProfile.aspx HYPERLINK "https://data.un.org/CountryProfile.aspx.crNam e=Kyrgyzstan". HYPERLINK "https://data.un.org/CountryProfile.aspx.crNam e=Kyrgyzstan"crName=Kyrgyzstan

2. (2011) World Health Organization. 2011. MDR-TB and XDR-TB 2011 Progress Report.

3. (2013) World Health Organization. Tuberculosis country work summary, Kyrgyzstan: Epidemiological profile. 2013

4. (2011) World Health Organization. Tuberculosis country work summary, Kyrgyzstan: Epidemiological profile. 2011

5. (2011) World Health Organization. Rapid implementation of the Xpert MTB/RIF diagnostic test; technical and operational 'howto' practical considerations. Geneva: WHO; 2011.

6. (2014) Modern TB Diagnostic Services: Optimizing the Old with the New. $6^{\text {th }}$ Global Laboratory Initiative Partners Meeting. WHO Stop TB Partnership. 2014.

7. Boehme CC et al. (2011) Feasibillity, diagnostic accuracy, and effectiveness of decentralized use of the Xpert MTB/RIF test for diagnosis of tuberculosis and multidrug resistance: a multicenter implementation study. Lancet 2011.

8. Steingart KR, Sohn H, Schiller I, Kloda LA, Boehme CC, Pai M, Dendukuri N. (2013). Xpert ${ }^{\circledR M T B / R I F}$ assay for pulmonary tuberculosis and rifampicin resistance in adults (Review). Cochrane Database of Systematic Reviews, Issue 1. Art. No.: CD009593. DOI: 10.1002/14651858.CD009593.pub2.

9. (2014) Summary of Clinical Protocols on Tuberculosis. Ministry of Health of Kyrgyz Republic. National Tuberculosis Center. Bishkek-2014.

10. Marlowe EM, Novak-Weekley SM, Cumpio J, Sharp SE, Momeny MA, Babst A, Carlson J, Kawamura M, Pandori M. (2011). Evaluation of the Cepheid Xpert MTB/RIF Assay for Direct Detection of Mycobacterium tuberculosis Complex in Respiratory Specimens. Journal of Clinical Microbiology.

11. Xichao Ou, Hui Xia, Qiang Li, Yu Pang, Shengfen Wang, Bing Zhao, Yuanyuan Song, Yang Zhou, Yang Zheng, Zhijian Zhang, Zhiying Zhang, Junchen Li, Haiyan Dong,
Junying Chi, Jack Zhang, Kai Man Kame, Shitong Huan, Yue Jun., Daniel P., Chin, Yanlin Zhao. (2018) A feasibility study of the Xpert MTB/RIF test at the peripheral level laboratory in China. International Journal of Infectious Diseases.

12. Boyles TH, Hughes J, Cox V, Burton R, Meintjes G, Mendelson M. (2014). Falsepositive Xpert MTB/RIF assays in previously treated patients: need for caution in interpreting results. International Journal of Tuberculosis and Lung Diseases.

13. Kampen SC, SusantoNH, SimonS, Astiti SD, Chandra R, Burhan E, et al. (2015) Effects of Introducing XpertMTB/RIF on Diagnosis and Treatment of Drug-Resistant Tuberculosis Patients in Indonesia: A Pre-Post Intervention Study. $\quad$ PLoSONE. 0(6):e0123536.doi:10.1371/journal.pone.01235 36

14. Narasimooloo R, Ross A. (2012) Delay in commencing treatment for MDR $\mathrm{TB}$ at a specialized TB treatment centre in KwaZuluNatal. South African Medical Journal. (2012). ISSN 0256-9574

15. (2012)

Retrieved from:http://www.scielo.org.za/scielo.php?pid=S 0256-95742012000600019 HYPERLINK "http://www.scielo.org.za/scielo.php?pid=S025 6-95742012000600019\&script=sci arttext"\& HYPERLINK

"http://www.scielo.org.za/scielo.php?pid=S025 6-

95742012000600019\&script=sci_arttext"script =sci arttext

16. Falzon D, Mirzayev F, Wares F et al. (2014) Multidrug-resistant tuberculosis around the world: what progress has been made? Global TB Programme, WHO, Geneva, Switzerland. (2014) ERJ Express: 10.1183

17. Lawn Stephen, et al. (2011). Screening for HIV-Associated Tuberculosis and Rifampicin Resistance before Antiretroviral Therapy Using the Xpert MTB/RIF Assay: A Prospective Study. Plos Medicine. 10: 1371.

18. Theron G, et al. (2011). Evaluation of the Xpert MTB/RIF assay for the diagnosis of pulmonary tuberculosis in a high HIV prevalence setting. Am. J. Respir. Crit. Care Med. 184:132- 140

19. Mokaddas E, Ahmad S, Eldeen HS, Al-Mutairi N. J Clin Microbiol. (2015) Apr;53(4):1351-4. doi: 10.1128/JCM.03412-14 